\title{
TRATAMIENTO BIOLÓGICO DE LIXIVIADOS DE RELLENOS SANITARIOS
}

Por:

Alexander Álvarez Contreras' ${ }^{1}$ John Hermógenes Suárez Gélvez²

\section{RESUMEN}

El trabajo realizado tuvo por finalidad la caracterización y cuantificación del lixiviado generado en el relleno sanitario "El Guayabal " de la ciudad San José de Cúcuta y la evaluación de dos sistemas de tratamiento biológico a escala laboratorio para este tipo de residuo.

La producción del lixiviado evaluado para el período de menor precipitación anual oscilaba entre 0,34 y $34,47 \mathrm{~m}^{3} / \mathrm{d}$ y para la época de mayor precipitación se obtuvo un caudal promedio de $48,34 \mathrm{~m}^{3} / \mathrm{d}$. La $\mathrm{DQO}$ del lixiviado se encontraba en un rango de 7.650 a $28.250 \mathrm{mg} / \mathrm{L}$. Los sistemas de tratamiento ensayados fueron: un reactor anaerobio del tipo UASB y un sistema de Biodiscos.

El reactor de biodiscos se construyó de una etapa con un volumen de $60 \mathrm{~L}$ y 48 discos. La carga máxima asimilada por el sistema fue de $31 \mathrm{gDQO} / \mathrm{m}^{2 *} \mathrm{~d}$, con una eficiencia de remoción promedio en $\mathrm{DQO}$ de $70 \%$ y eficiencias de remoción máximas por encima del $90 \%$ para una carga optima en un rango de 15 a 20 $\mathrm{gDQO} / \mathrm{m}^{2 *} \mathrm{~d}$, además este sistema presentó gran estabilidad frente a variaciones importantes en cuanto a las características del lixiviado y ausencia de olores desagradables.

El reactor Anaerobio (UASB) de 39,1 L de volumen se operó con un tiempo de retención hidráulica de 36 horas. Los porcentajes de remoción de DQO en el reactor UASB eran muy variables, con valores muy bajos $(5,13 \%)$ para una COV de $3.91 \mathrm{KgDQO} / \mathrm{m}^{3 *} \mathrm{~d}$ y eficiencias representativas para condiciones óptimas de operación $(65,45 \%)$ y COV de 0,91 KgDQO/m²d, lo cual indica que el sistema no asimilaba cargas volumétricas altas durante el periodo de experimentación, esto se debió principalmente a que no se dieron las estrictas condiciones para este sistema.

Palabras claves: Lixiviado, Biodiscos, UASB, DQO.

\section{ABSTRACT}

The carried out work had for purpose the characterization and quantification of the one leached generated in the land-fill "El Guayabal" of San José of Cúcuta city and the evaluation of two systems of biological treatment to scale laboratory for this residual type.

The production of the one leached evaluated for the period of smaller annual precipitation oscillated between 0,34 and $34,47 \mathrm{~m}^{3} / \mathrm{d}$ and for the time of more precipitation a flow average of $48,34 \mathrm{~m}^{3} / \mathrm{d}$ was obtained. The COD of the one leached was in a range from 7.650 to $28.250 \mathrm{mg} / \mathrm{L}$. The treatment tried systems were: an UASB type anaerobic reactor, and one Rotating Biological Contactor system.

The Rotating Biological contactor was built of a stage with a volume of $60 \mathrm{~L}$ and 48 disks. The biggest load adsorbed by Rotating Biological contactor system was $31 \mathrm{~g} \mathrm{COD} / \mathrm{m}^{2 *} \mathrm{~d}$, with an average of removal efficiency about COD of $70 \%$; and greatest removal efficiency over $90 \%$ for a very best load in one rank from 15 to $20 \mathrm{~g} \mathrm{COD} / \mathrm{m}^{2 *} \mathrm{~d}$, moreover this system presented great stability in front of important variations on leached characteristics and absence of disagreeable faint odors.

The UASB reactor of $39,1 \mathrm{~L}$ of volume was operated with a time of hydraulic retention of 36 hours. The

Ingeniero de Producción Biotecnológica. Asistente de Laboratorio de Aguas. Integrante Grupo de Investigaciones Ambientales (GUIA) Universidad Francisco de Paula Santander. alexanderalvarezcontreras@yahoo.es

2 Ingeniero Químico. Esp. En Ingeniería Sanitaria y Ambiental, estudiante de MSc en Ingenieria Sanitaria y Ambiental. Docente de la Facultad de Ciencias Agrarias y del Ambiente. Universidad Francisco de Paula Santander. johnhermogenes@yahoo.com. 
Tratamiento biológico de lixiviados de rellenos sanitarios

percentages of removal of COD in the UASB reactor were very variable, with very low values $(5,13 \%)$ for a COV of $3.91 \mathrm{Kg} \mathrm{COD} / \mathrm{m}^{3 *} \mathrm{~d}$ and representative efficiencies for good conditions of operation $(65,45 \%)$ and COV of 0,91 $\mathrm{KgCOD} / \mathrm{m}^{3 *}$, that which indicates that the system didn't assimilate volumetrical loads high during the period of experimentation, this was principally due to that the strict system conditions weren't done.

Key words: Leached, Rotating Biological Contactor, UASB, COD.

\section{INTRODUCCIÓN}

Los lixiviados son todos aquellos líquidos que han entrado en contacto con los desechos de rellenos sanitarios y se producen por la disolución de uno o más compuestos de los residuos sólidos urbanos en contacto con el agua, o por la propia dinámica de descomposición de los residuos.

El lixiviado generado en un Relleno Sanitario es función de múltiples factores, tales como: composición de la basura, edad del Relleno, balance de agua, diseño y operación del Relleno sanitario, solubilidad de los desechos, procesos de conversión microbiológica y química y la interacción del lixiviado con el medio ambiente. El caudal generado y la composición varían de acuerdo al estado de avance y el tipo de operación del relleno[1].

Existen varios antecedentes de tratamiento aerobio y anaerobio de lixiviados, que van desde experiencias a escala laboratorio a experiencias a escala real. El tipo de tratamiento aerobio mas extendido es lodos activados o lagunas aireadas[2]. Otro sistema aerobio utilizado para el tratamiento de los lixiviados es el reactor de biodiscos o CBR (Contactor Biológico Rotante). En cuanto al tratamiento anaerobio de lixiviado, el sistema de mayor difusión es el reactor UASB. Las experiencias reportadas ofrecen resultados que favorecen al tratamiento anaerobio de lixiviados, pero en diferentes casos presentan problemas específicos que dependen del lixiviado que se trata [3]. Las características fisicoquímicas del lixiviado varían de un lugar a otro, por lo que es necesario realizar estudios para cada caso en particular.

El relleno sanitario "El Guayabal" se encuentra ubicado en la vereda Guayabal, aproximadamente a unos $10 \mathrm{Km}$ al norte de la ciudad San José de Cúcuta, recibe residuos sólidos domiciliarios del Área Metropolitana y de varios municipios del Norte de Santander. Se disponen en promedio 473,99 toneladas por día.[4]

Los lixiviados generados en el Relleno Sanitario "El Guayabal" alcanzan caudales que oscilan entre 6.91 y $17.28 \mathrm{~m}^{3} / \mathrm{d}$ en los tres años de vida útil que tiene hasta la fecha. Estos lixiviados son capturados mediante un sistema conformado por tuberías perforadas y almacenados en una laguna de evaporación, para luego ser recirculados a las celdas de operación del Relleno Sanitario sin tratamiento alguno[4]. En el presente trabajo se realizó un diagnóstico de calidad y cantidad del lixiviado generado en el Relleno Sanitario "El Guayabal", y se ensayó con un sistema de tratamiento aerobio y uno de tratamiento anaerobio, analizándose ventajas y desventajas de cada uno de ellos.

\section{MATERIALES Y MÉTODOS}

Monitoreo de Calidad y Cantidad del Lixiviado.

Debido a que el diseño de una planta de tratamiento de lixiviados esta directamente condicionada al caudal y a las características del lixiviado generado en el relleno sanitario, en esta investigación se realizaron campañas de aforo, muestreo y caracterización al lixiviado generado en el Relleno sanitario "El Guayabal". Los monitoreos se realizaron una vez por mes durante el periodo comprendido entre los meses de Julio a Noviembre del año 2003, se tomaron muestras de tipo compuestas. Las características analizadas al lixiviado se citan en la tabla 1. 
Tratamiento biológico de lixiviados de rellenos sanitarios

Tabla 1. Variables analizadas al lixiviado.

\begin{tabular}{|c|c|}
\hline ANÁLISIS & MÉTODO \\
\hline DBO5 & Winkler \\
DQO & Colorimetría \\
Nitratos & Colorimetría \\
Nitritos & Colorimetría \\
Amonio & Colorimetría \\
Sulfatos & Colorimetría \\
Fosfatos & Colorimetría \\
Sólidos totales & Gravimétrico \\
Sólidos Suspendidos Totales & Gravimétrico \\
Sólidos Suspendidos Volátiles & Gravimétrico \\
Sólidos Sedimentables & Volumétrico \\
Acidez & Volumétrico \\
Alcalinidad & Volumétrico \\
Cloruros & Volumétrico \\
Dureza Total & Volumétrico \\
Temperatura & Electrométrico \\
pH & Electrométrico \\
Metales & Absorción Atómica \\
Microbiológicos & Placa Profunda \\
\hline
\end{tabular}

Las determinaciones se realizaron en el Laboratorio de Aguas de la Universidad Francisco de Paula Santander (U.F.P.S.), de acuerdo con las técnicas del Standard Methods (APHA, AWWA, WEF, 1995) y a los protocolos publicados por el IDEAM. (disponibles en la pagina web de este instituto)

El lixiviado utilizado para el arranque y operación de los reactores se tomaba del tanque colector más cercano a la laguna de evaporación con una frecuencia semanal.

\section{Arranque y Operación de los sistemas a escala} laboratorio.

Los reactores fueron instalados en un área física, ubicada dentro del relleno sanitario, la cual fue adecuada para que reuniera las condiciones necesarias para el desarrollo de la investigación.

\section{Sistema de Biodiscos.}

Se construyó un reactor aerobio tipo Biodiscos de 60 litros de volumen, elaborado en material plástico, compuesto por 48 discos de acetato cada uno con un diámetro de $30 \mathrm{~cm}$, que se lijaron para facilitar la adhesión de la biomasa. El sistema funcionó a una velocidad de 5,5 rpm. El reactor se inoculó con lodo proveniente de un digestor aerobio que trata aguas residuales domesticas de un instituto de investigación en petróleo, ubicado en Bucaramanga, Santander. Se adicionaron al reactor 36 litros de lodo con una relación STV/ST de 0,96, teniendo en cuenta la experiencia llevada acabo por Dautant y López (1999)[5].

El sistema se alimentó con lixiviado puro del tanque colector de lixiviados más cercano a la laguna de evaporación. El arranque del sistema fue realizado con aumentos sucesivos de carga orgánica superficial (COS), establecida por la siguiente ecuación[6]:

$$
\operatorname{COS}=\frac{Q \times D Q O}{A}
$$

Donde:

- COS: Carga Orgánica Superficial (g DQO $/ \mathrm{m}^{2 *} d$ )

- Q: Caudal (L/d)

- DQO: Concentración de DQO del afluente (g $\mathrm{DQO} / \mathrm{L})$

- A: Área de los discos $\left(6.7858 \mathrm{~m}^{2}\right)$.

Las condiciones operacionales se muestran en la siguiente tabla 2 :

Tabla 2. Parámetros de operación para el sistema de Biodiscos.

\begin{tabular}{|c|c|c|}
\hline $\begin{array}{c}\text { COS } \\
\left(\mathrm{g} D Q O / \mathrm{m}^{2 *} \mathrm{~d}\right)\end{array}$ & $\begin{array}{c}\text { Caudal } \\
(\mathrm{ml} / \mathrm{min})\end{array}$ & $\begin{array}{c}\text { Periódo de } \\
\text { incrementos de } \\
\text { carga (semanas) }\end{array}$ \\
\hline 1 & 0.62 & 1 \\
\hline 3 & 2.26 & 1 \\
\hline 5 & 4.17 & 2 \\
\hline 10 & 5.96 & 2 \\
\hline 20 & 8.05 & 2 \\
\hline 30 & 11.45 & 1 \\
\hline
\end{tabular}

El criterio para el aumento en la carga fue el comportamiento del sistema en cuanto a remoción de DQO. 
Tratamiento biológico de lixiviados de rellenos sanitarios

El seguimiento del reactor se realizó mediante la determinación de Caudal, pH, Temperatura, DQO, SST, SSV, Nitrógeno amoniacal, Alcalinidad y oxígeno disuelto. Las determinaciones se realizaron de acuerdo a técnicas del Standard Methods (APHA, AWWA, WEF, 1995) y a los protocolos publicados por el IDEAM (disponibles en la pagina web de este instituto).

\section{Reactor UASB.}

Se trabajó con un reactor del tipo manto de lodos anaerobio de flujo ascendente UASB, elaborado en tubería PVC de 6 pulgadas de diámetro, con un volumen de 39, 1 litros y operado con un TRH constante de 36 horas[3].

El reactor fue inoculado (aproximadamente un tercio del volumen, 13 litros) con lodo proveniente de un reactor anaerobio UASB empleado para el tratamiento de las aguas residuales generadas por una fábrica embotelladora de bebidas gaseosas, previamente al arranque del reactor. Al lodo se le realizaron mediciones de $\mathrm{pH}$, sólidos suspendidos totales y volátiles y se hicieron ensayos de sedimentabilidad y Actividad Metanogénica Específica para determinar la calidad del inóculo.

El arranque del reactor se hizo con el criterio de aumento de concentración de DQO. Se comenzó con una concentración teórica de $1500 \mathrm{mgDQO} / \mathrm{L}$ ( $1,0 \mathrm{kgDQO} / \mathrm{m}^{3}$.día de COV), esto teniendo en cuenta la experiencia llevada a cabo en Cali en el Relleno Transitorio de Navarro[3].

El criterio para aumentar la concentración de DQO para el periodo de operación fue la respuesta del reactor en términos de eficiencia de remoción de $\mathrm{DQO}$ y reducción de AGV. El manejo de las concentraciones de DQO en el afluente, se llevó a cabo mediante diluciones, ya que el lixiviado presentaba altas concentraciones de DQO.

El seguimiento del reactor se realizó mediante determinación de caudal, pH, Temperatura, DQO, AGV, SST, SSV, Alcalinidad y nitrógeno amoniacal. Las determinaciones se realizaron de acuerdo a técnicas del Standard Methods (APHA, AWWA, WEF, 1995) y a los protocolos publicados por el IDEAM (disponibles en la página web de este instituto).

\section{RESULTADOS Y DISCUSIONES}

Monitoreo de Calidad y Cantidad del Lixiviado. Los caudales del lixiviado del Relleno sanitario generalmente fueron estables en los primeros meses de muestreo, que correspondieron a época de verano donde se registraban temperaturas ambientales hasta de $39^{\circ} \mathrm{C}$ y temperaturas del lixiviado de $45^{\circ} \mathrm{C}$. La época de invierno comenzó en los primeros días de Noviembre, incrementándose el caudal por las lluvias frecuentes y por las recirculaciones periódicas de lixiviado al sitio de disposición final de Residuos sólidos. (Ver tabla 3).

Tabla 3. Caudales de lixiviado del relleno sanitario "El Guayabal".

\begin{tabular}{|l|c|c|c|c|c|}
\hline Caudal $\left(\mathrm{m}^{3} / \mathrm{d}\right)$ & Julio & Agosto & Septiembre & Octubre & Noviembre \\
\hline Máximo & 15.21 & 34.47 & 6.48 & 8.99 & 186.45 \\
\hline Mínimo & 0,34 & 4.32 & 1.81 & 1.73 & 4.32 \\
\hline Promedio & 7.83 & 8.31 & 4.73 & 5.70 & 48.34 \\
\hline
\end{tabular}

La DQO del lixiviado del tanque colector presentaba un rango de 7.650 - $28.250 \mathrm{mg} / \mathrm{L}$, valor que se encuentra dentro del rango reportado para lixiviados producidos en un relleno sanitario con una edad igual o menor a 2 años, como es el caso del Relleno Sanitario "El Guayabal", en los días de muestreo. (Ver tabla 4).

La relación $\mathrm{DBO}_{5} / \mathrm{DQO}$ del lixiviado del tanque colector se encontraba en un rango de $0.68-0.89$, y con un promedio de 0,78 . Este rango indica que la materia orgánica de los lixiviados es fácilmente biodegradable[7].

El pH un poco alto se atribuye a la alcalinidad producida por una elevada concentración de nitrógeno amoniacal 
Tratamiento biológico de lixiviados de rellenos sanitarios

lo que concede una alta capacidad buffer, pero este rango de $\mathrm{pH}$ y concentraciones altas de nitrógeno amoniacal origina una inhibición del proceso anaerobio.

La concentración de metales pesados en este lixiviado son importantes, principalmente el cromo el cual proviene de residuos de curtiembres que se depositan en este relleno.

En general las características de este lixiviado varían a través del tiempo, y esto se debe a las diluciones con el agua lluvia y procesos de degradación de la materia orgánica entre otros factores.

Tabla 4. Características Fisicoquímicas del lixiviado del relleno sanitario "El Guayabal".

\begin{tabular}{|c|c|c|c|c|}
\hline PARAMETRO & UNIDAD & PROMEDIO & MÍNIMO & MAXIMO \\
\hline $\mathrm{pH}$ & - & 7,54 & 6,5 & 8,35 \\
\hline DQO & $\mathrm{mg} / \mathrm{L} \mathrm{O}_{2}$ & 16334 & 7650 & 28250 \\
\hline $\mathrm{DBO}_{5}$ & $\mathrm{mg} / \mathrm{L} \mathrm{O}_{2}$ & 12878 & 5250 & 20890 \\
\hline $\mathrm{DBO}_{5}: \mathrm{DQO}$ & & 0,78 & 0,68 & 0,89 \\
\hline Sólidos totales & $\mathrm{mg} / \mathrm{L}$ & 15961 & 11478 & 24370 \\
\hline Sólidos sedimentables & $\mathrm{ml} / \mathrm{L}^{*} \mathrm{~h}$ & 1,2 & 0,2 & 3 \\
\hline Sólidos suspendidos & $\mathrm{mg} / \mathrm{L}$ & 2043,2 & 336 & 3974 \\
\hline Turbiedad & NTU & 359 & 210 & 600 \\
\hline Cloruros & $\mathrm{mg} / \mathrm{L} \mathrm{Cl}^{-}$ & 1496 & 260 & 5300 \\
\hline Dureza total & $\mathrm{mg} / \mathrm{L} \mathrm{CaCO}_{3}$ & 3878 & 1764 & 5750 \\
\hline Alcalinidad total & $\mathrm{mg} / \mathrm{l} \mathrm{CaCO} 3$ & 6875 & 2750 & 9500 \\
\hline Nitrógeno Total & $\mathrm{mg} / \mathrm{L} \mathrm{N}$ & 1266,6 & 761,45 & 1959,72 \\
\hline Nitrógeno amoniacal & $\mathrm{mg} / \mathrm{L} \mathrm{NH}_{3}$ & 809,25 & 577,5 & 937 \\
\hline Nitratos & $\mathrm{mg} / \mathrm{L} \mathrm{NO}_{3}^{-}$ & 107,31 & 60,48 & 215 \\
\hline Nitritos & $\mathrm{mg} / \mathrm{L} \mathrm{NO}_{2}^{-}$ & 1,39 & 0,54 & 1,98 \\
\hline Fosfatos & $\mathrm{mg} / \mathrm{LPO}_{4}^{-3}$ & 30,03 & 24,30 & 34,75 \\
\hline Sulfatos & $\mathrm{mg} / \mathrm{L} \mathrm{SO}_{4}^{-2}$ & 120,67 & 50 & 175 \\
\hline Cobre & $\mathrm{mg} / \mathrm{L} \mathrm{Cu}$ & 0,04 & 0,008 & 0,064 \\
\hline Plomo & $\mathrm{mg} / \mathrm{L} \mathrm{Pb}$ & 0,032 & 0,021 & 0,041 \\
\hline Cromo & $\mathrm{mg} / \mathrm{L} \mathrm{Cr}$ & 0,316 & 0,034 & 0,861 \\
\hline Zinc & $\mathrm{mg} / \mathrm{L} \mathrm{Zn}$ & 0,75 & 0,37 & 1,70 \\
\hline Litio & $\mathrm{mg} / \mathrm{L} \mathrm{Li}$ & 0,19 & 0,15 & 0,33 \\
\hline Manganeso & $\mathrm{mg} / \mathrm{L} \mathrm{Mn}$ & 12,82 & 0,17 & 35,40 \\
\hline Hierro total & $\mathrm{mg} / \mathrm{L} \mathrm{Fe}$ & 42,23 & 23,37 & 77,8 \\
\hline Potasio & $\mathrm{mg} / \mathrm{LK}$ & 2045,6 & 504,2 & 4048 \\
\hline Sodio & $\mathrm{mg} / \mathrm{L} \mathrm{Na}$ & 1976,92 & 919,8 & 3991 \\
\hline Calcio & $\mathrm{mg} / \mathrm{L} \mathrm{Ca}$ & 655 & 200 & 1300 \\
\hline Magnesio & $\mathrm{mg} / \mathrm{L} \mathrm{Mg}$ & 639 & 235 & 928 \\
\hline Coliformes totales & $\mathrm{ufc} / \mathrm{ml}$ & 53900 & 35600 & 80000 \\
\hline Coliformes fecales & $\mathrm{ufc} / \mathrm{ml}$ & 14125 & 3500 & 28000 \\
\hline Hongos y levaduras & $\mathrm{c} / \mathrm{ml}$ & 11000 & 2000 & 1050000 \\
\hline
\end{tabular}

Fuente: ÁlVAREZ CONTRERAS Alexander. Diseño de una planta de tratamiento a escala Piloto para el lixiviado generado en el relleno sanitario "El Guayabal" de la ciudad San José de Cúcuta. San José de Cúcuta, Universidad Francisco de Paula Santander. 2004.

\section{Arranque y Operación de los Sistemas.}

\section{Sistema de Biodiscos}

El aumento de la carga orgánica superficial (COS), se realizaba con el objetivo de encontrar la carga óptima asimilada por el sistema. Se comenzó el arranque con una carga de $1 \mathrm{~g} \mathrm{DQO} / \mathrm{m}^{2 *} \mathrm{~d}$, a medida que se observaba una adaptación del sistema a las condiciones del lixiviado, se aumentaba la carga orgánica superficial. Las oscilaciones en los valores de la carga se deben a dificultades en la regulación del caudal y a obstrucciones en las conexiones. (Ver figura 1).

Figura 1. Relación COS - Eficiencia del sistema Aerobio Reactor de Biodiscos.

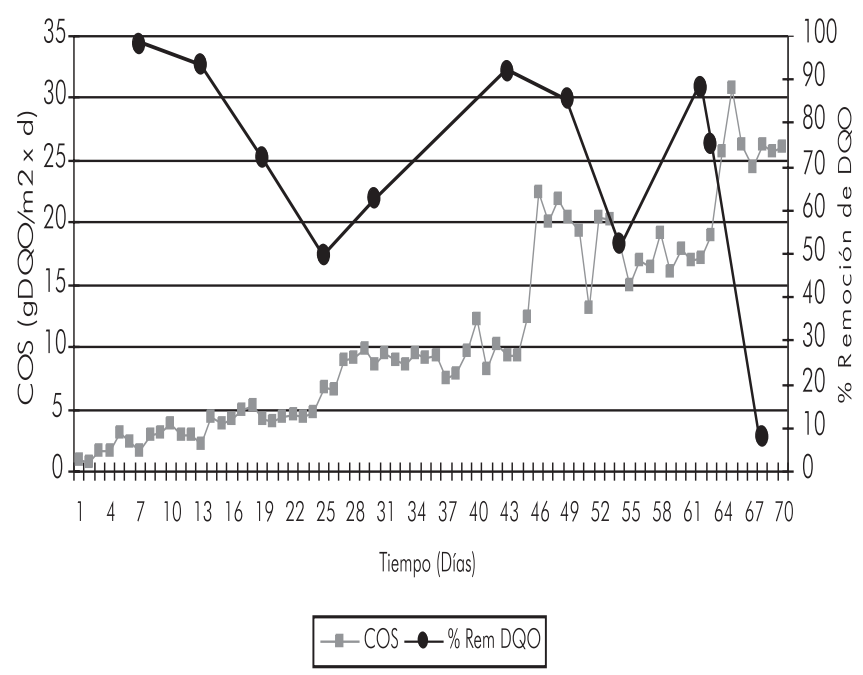

Este sistema se caracterizaba por una alta eficiencia en los primeros días de operación, estos valores de eficiencia no son representativos para el estudio, ya que en el arranque del reactor se utilizaron aguas blancas y lodo de inóculo, que producían una dilución en la concentración de los lixiviados y las cargas que se inyectaban al sistema eran muy bajas, esto se hizo con el objetivo de que el lodo se adaptara poco a poco a las condiciones del lixiviado. (Ver figura 1)

Entre los días 1 al 25, se observa un descenso en la eficiencia de remoción, esto se atribuye a que en este 
Tratamiento biológico de lixiviados de rellenos sanitarios

periodo la película biológica se encontraba en proceso de formación y los microorganismos presentes en ella, estaban empezando adaptarse a las condiciones del residuo.

Se puede observar que la carga óptima del sistema se encontraba en un rango de 15 a $20 \mathrm{gDQO} / \mathrm{m}^{2 *}$, donde se obtuvo los mayores valores en porcentaje de remoción en DQO. Cuando el sistema se mantenía en el rango anteriormente mencionado, el porcentaje de remoción se conservaba con valores mayores de $52,29 \%$, presentando su máximo valor $(91,91 \%)$ el día 43 durante el periodo de arranque y operación. Estos resultados fueron similares a los reportados por Borzacconi, López y Arcia (1996)[2].

El día 42 se presentó una disminución en la película biológica, que puede deberse al efecto cortante de los discos con la superficie del agua, que produce una caída del material celular muerto. La película biológica se recuperó de una manera rápida, demostrando la gran estabilidad de este sistema a condiciones desfavorables tales como, condiciones alcalinas de $\mathrm{pH}$, concentraciones elevadas del afluente, aumentos de la carga orgánica y resistencia a los compuestos tóxicos presentes en el lixiviado.

\section{Reactor UASB.}

La caracterización del lodo utilizado como inóculo se muestra en la tabla 5.

Tabla 5. Caracterización del Inóculo (Lodo Reactor UASB Embotelladora de Bebidas Gaseosas)

\begin{tabular}{|c|c|c|}
\hline PARÁMETRO & RESULTADO & UNIDADES \\
\hline SST & 70.76 & $\mathrm{~g} / \mathrm{L}$ \\
\hline SSV & 64.82 & $\mathrm{~g} / \mathrm{L}$ \\
\hline SSV/SST & 0.91 & - \\
\hline AME & 0,13 & $\mathrm{gDQO}_{\text {CH4 }} / \mathrm{gSSV}$.día \\
\hline Sedimentabilidad & 2,82 & $\mathrm{~m} / \mathrm{h}$ \\
\hline
\end{tabular}

Los resultados obtenidos en las pruebas de Actividad Metanogénica Especifica, Sedimentabilidad y Sólidos suspendidos, indican que el lodo tenía regulares características para ser utilizado como inóculo, comparándolo con un lodo granular para tratar residuos con alta carga orgánica[8], teniendo en cuenta que las concentraciones de DQO en este lixiviado eran muy elevadas. Por lo cual se determinó realizar el arranque con concentraciones de DQO bajas por un largo periodo hasta que los microorganismos presentes en lodo se adaptaran a este tipo de residuo.

La figura 2, muestra que el porcentaje de remoción de DQO en el reactor UASB era muy variable, con picos bajos y caídas muy marcadas, que indican que no se presentaba un funcionamiento óptimo del proceso durante la mayor parte del periodo de operación del reactor UASB. Esta situación se pudo presentar por las siguientes razones:

- La inhibición causada por el amoniaco en el proceso de metanogénesis.

- Las concentraciones de DQO del afluente no correspondían a las calculadas en la ecuación $C_{1} V_{1}=C_{2} V_{2}$, por lo tanto se inyectaban concentraciones de DQO muy elevadas al sistema.

- Las condiciones ambientales de $\mathrm{pH}$ alcalino no permitían el buen funcionamiento del proceso anaerobio, ya que el $\mathrm{pH}$ óptimo para procesos anaerobios $(6,8$ a 7,2$)[8]$. Se pudo mantener solo en los últimos días de la experiencia.

- Las concentraciones altas de AGV durante el arranque del reactor, pudieron ocasionar una inhibición de los microorganismos metanogénicos presentes en el lodo.

- El balance nutricional proporcionado por el lixiviado, no era el ideal para el tratamiento anaerobio, ya que en la caracterización fisicoquímica del lixiviado, se pudo observar una deficiencia de fósforo. 
Tratamiento biológico de lixiviados de rellenos sanitarios

La figura 2, muestra definitivamente un regular desempeño del reactor cuando el $\mathrm{pH}$ afluente se presentaba mayor a 8 unidades (entre los días 1 y 53), durante este periodo se obtuvieron porcentajes de remoción de DQO menores de 55,86\%. Después de ajustar el $\mathrm{pH}$ a un rango de 6,8 - 7,2 unidades en el afluente para eliminar la inhibición ocasionada por el amoniaco, entre los días 54 y 63, se notó un importante aumento $(65,45 \%)$ en el porcentaje de remoción en $\mathrm{DQO}$, que se atribuye a la adaptación de los microorganismos a las nuevas condiciones del afluente.

Figura 2. Comportamiento del reactor UASB

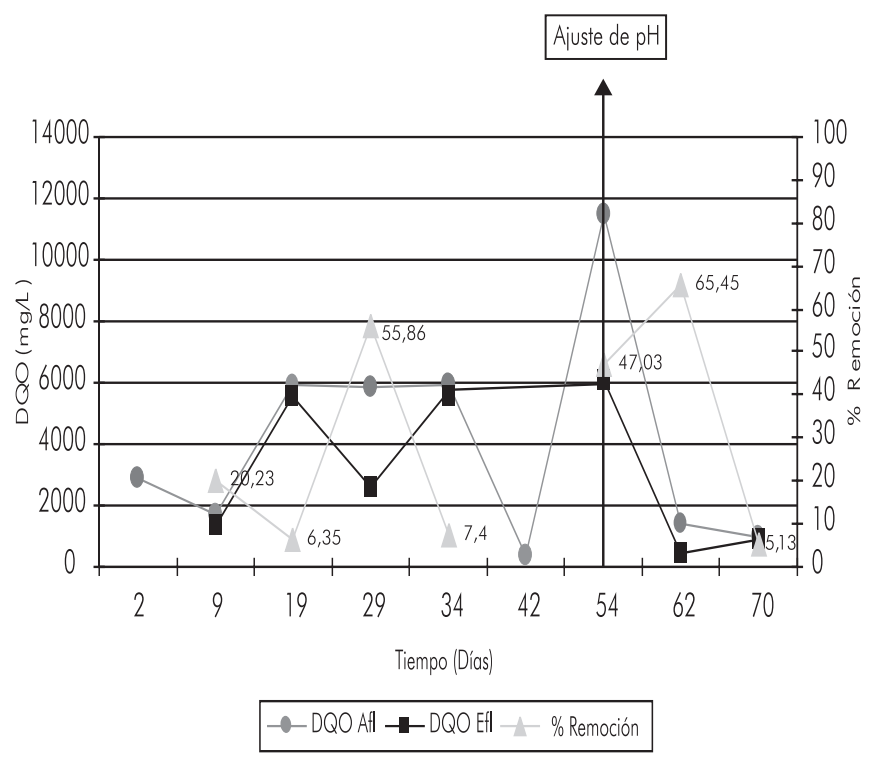

La alcalinidad varió de manera significativa en el transcurso de la operación del sistema, la concentración de alcalinidad total en el efluente presentó los mayores valores en los primeros días de la fase experimental, el día 42 presentó una disminución significativa en la concentración de la alcalinidad total del efluente, esto se dio por que el lixiviado que se inyectó en estos días presentaba una baja alcalinidad, que se pudo deber a la dilución del lixiviado realizada para alimentar el sistema en los días anteriores a este análisis.
Los elevados valores de alcalinidad garantizan la capacidad del sistema para neutralizar los AGV producidos, pues al tener una alcalinidad Total mayor a $750 \mathrm{mgCaCO} 3 / \mathrm{L}$, se garantiza que los AGV no son tóxicos ya que se encuentran en forma de sales reduciendo su toxicidad hasta un $75 \%$.

En la figura 3, se muestra que en algunos días, la alcalinidad presentaba una concentración mayor en el efluente que el afluente del reactor UASB, esto puede ser a causa de una acumulación de amoniaco en el sistema, el cual generó una alcalinidad adicional contribuyendo así a mantener las características alcalinas de $\mathrm{pH}$.

Figura 3. Relación AGV y Alcalinidad Total del Sistema Anaerobio (Reactor UASB).

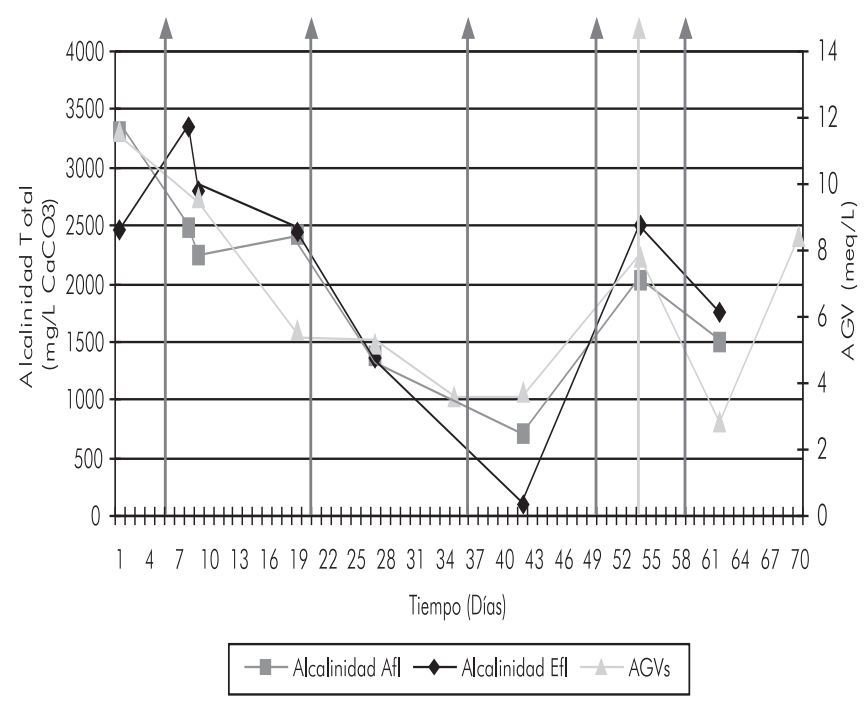

\section{CONCLUSIONES}

De acuerdo al monitoreo de calidad y cantidad del lixiviado generado en el relleno sanitario "El Guayabal". El caudal del lixiviado, esta en función de muchos factores tales como las condiciones metereológicas del área de influencia, la cantidad y composición de los residuos sólidos dispuestos en el relleno Sanitario, las actividades propias de la operación del relleno sanitario, entre otros factores. 
Tratamiento biológico de lixiviados de rellenos sanitarios

El sistema de biomasa fija tipo Biodiscos, presentó buena eficiencia de remoción y gran estabilidad frente a variaciones importantes en la composición del lixiviado y en la carga orgánica. La carga óptima del reactor de Biodiscos, se encontró en un rango de 10 a $20 \mathrm{gDQO} / \mathrm{m}^{2 *} \mathrm{~d}$, este fue el rango en el cual el sistema de Biodiscos alcanzó los más altos valores en porcentaje de remoción de DQO con un promedio de $75,88 \%$, un valor máximo de $91,91 \%$ con una de DQO en el afluente de $11.750 \mathrm{mg} / \mathrm{l}$ y en el efluente de $950 \mathrm{mg} / \mathrm{l}$ y un valor mínimo de 52,29\%, para valores de DQO de $9.275 \mathrm{mg} / \mathrm{l}$ y $4.425 \mathrm{mg} / \mathrm{l}$ en el afluente y efluente respectivamente.

Los resultados de porcentajes de remoción reportados para el Sistema Anaerobio Reactor UASB, no alcanzaron un nivel alto esperado, ya que obtuvieron valores menores de 65,45 \%, a concentraciones bajas de DQO. Sin embargo, es necesario resaltar la necesidad de controlar el $\mathrm{pH}$ del lixiviado en el afluente, en este caso particular el lixiviado posee un alto contenido de Nitrógeno amoniacal y como consecuencia de un aumento de $\mathrm{pH}$ se puede alcanzar concentraciones inhibitorias de amoniaco libre.

De acuerdo con los resultados obtenidos en el transcurso de la operación del reactor UASB, la selección del lodo de inóculo es un factor importante en el proceso de degradación anaeróbia, no sólo se debe tener presente la procedencia del lodo, sino también sus características de Sedimentabilidad, concentración de SST y SSV, la Actividad Metanogénica Específica y la relación SSV/SST, ya que estos parámetros definen la calidad del inóculo.

La alcalinidad y el alto contenido de nitrógeno amoniacal, son factores que influyeron considerablemente en el comportamiento del proceso de tratamiento anaerobio evaluado, ya que el nitrógeno amoniacal ocasionó inhibición a las bacterias metanogénicas.
La tecnología UASB y Biodiscos no pueden ser usada como único sistema de tratamiento de lixiviados, ya que el efluente del tratamiento, aún conserva altas concentraciones de carga contaminante.

\section{AGRADECIMIENTOS}

La investigación fue realizada por la Facultad de Ciencias Agrarias y del Ambiente de la Universidad Francisco de Paula Santander (U.F.P.S.) gracias al apoyo técnico y económico de la Empresa ASEO URBANO S.A. E.S.P., la cual presta el servicio de recolección en un amplio sector del Area Metropolitana de Cúcuta y opera el relleno sanitario "El Guayabal".

\section{REFERENCIAS BIBLIOGRÁFICAS}

[1] PINEDA, Samuel Ignacio. Manejo y Disposición de Residuos Sólidos. Bogotá: Panamericana, 1998. 351 p.

[2] BORZACCONI Liliana, LOPEZ Ivan, ARCIA Esteban, CARDELINA Luis, CASTAGNA Alvaro, VIÑAS María. Comparación de Tratamientos Aerobios y Anaerobios Aplicados a Lixiviado de Relleno Sanitario. CEPIS OMS-OPS. 1996.

[3] MORAN SILVA, Adriana y NARVÁEZ MARTINEZ, Jorge. Evaluación del Comportamiento de dos Sistemas de Tratamiento Anaerobio para la Depuración de los Lixiviados Generados en El Relleno Transitorio de Navarro. Santiago de Cali: Univalle, 2002. 254p.

[4] ÁlVAREZ CONTRERAS Alexander. Diseño de una planta de tratamiento a escala Piloto para el lixiviado generado en el relleno sanitario "El Guayabal" de la ciudad San José de Cúcuta. San José de Cúcuta, Universidad Francisco de Paula Santander. 2004. 200 p.

[5] DAUTANT SEMPRUM, Rafael, LÓPEZ HERRERA, Hernán. Relación entre resultados en un reactor a escala laboratorio y otro a escala natural (caso real). CEPIS OMS-OPS. 1999. 
Tratamiento biológico de lixiviados de rellenos sanitarios

[6] ROMERO ROJAS, Jairo. Tratamiento de Aguas Residuales, Teoría y Principios de Diseño. Bogotá: Escuela Colombiana de Ingeniería, 2000. 317p.

[7] TCHOBANOGLOUS, George; THEISSEN, Hilary y VIGIL, Samuel. Gestión integral de residuos sólidos. Madrid: Mc Graw Hill, 1994. Tomo 1. 436p.

[8] UNIVERSIDAD DEL VALLE; CORPORACIÓN DEL VALLE DEL CAUCA y UNIVERSIDAD AGRÍCOLA WAGENINGEN. Arranque y operación de sistemas de flujo ascendente con manto de lodo UASB. Santiago de Cali: Univalle, 1987. $221 \mathrm{p}$.

Fecha recibido: Abril 24 de 2006

Fecha aceptación: Junio 15 de 2006 